\title{
La generación de capital social en el sistema educativo no universitario: un análisis del desarrollo comunitario de las escuelas de Cataluña
}

\author{
Julio Meneses \\ Josep M. Mominó \\ Universitat Oberta de Catalunya. Estudis de Psicologia i Ciències de l'Educació \\ Rambla del Poble Nou, 156. 08018 Barcelona (Spain). \\ jmenesesn@uoc.edu
}

\section{Resumen}

La presente investigación tiene como objetivo el estudio de los fenómenos comunitarios en el contexto del sistema educativo no universitario de Cataluña. Para ello, tras revisar los resultados de algunas aproximaciones realizadas anteriormente, se presenta un modelo de análisis basado en la teoría del capital social. De acuerdo con la distinción fundamental entre el capital social vinculante y aquél que tiende puentes, así como con la consideración de los diferentes ámbitos de actividad cotidiana de las escuelas (el aula y la escuela como contextos, así como sus respectivas relaciones con la comunidad local), se presentan los resultados obtenidos a partir de una muestra representativa de centros de educación primaria y secundaria de Cataluña. Para concluir, se discute la necesidad de considerar al propio desarrollo comunitario en el contexto escolar, no como un instrumento, sino como un fin en sí mismo.

Palabras clave: escuela, desarrollo comunitario, capital social, actividad cotidiana, participación, colaboración, calidad en la educación, eficacia escolar.

Abstract. Social capital building in the non-university education system: a community development analysis in Catalonian schools

The present research intends to study the community phenomena in the context of the non-university, Catalonian education system. Therefore, once the results of certain previous approximations have been revised, we present an analysis model based on the social capital theory. Together with the fundamental distinction between bonding and bridging social capital, and with the relevance of the varied circumstances of everyday life in schools (the classroom and the school contexts, and their respective connections to the local community), we present results from a representative sample of primary and secondary education centers from Catalonia (Spain). To conclude, we discuss the necessity to consider the community building in the schools not as an instrument, but as an end itself.

Key words: school, community building, social capital, everyday life, participation, collaboration, quality education, school effectiveness. 


\section{Sumario}

\section{Introducción 5. Metodología}

2. El concepto de comunidad: 6. Resultados de la contigüidad espacial a la construcción del «nosotros»

3. Una aproximación inicial al desarrollo comunitario del contexto escolar de Cataluña

4. La generación de capital social en el sistema educativo no universitario

\section{Discusión}

8. A modo de conclusión: el desarrollo comunitario como fin y no meramente como medio

Agradecimientos

Referencias bibliográficas

There is no obvious social motive for the acquirement of mere learning, there is no clear social gain in success thereat. Indeed, almost the only measure for success is a competitive one, in the bad sense of that term - a comparison of results [...] to see which child has succeeded in getting ahead of others in storing up, in accumulating the maximum of information. So thoroughly is this the prevalent atmosphere that for one child to help another in his task has become a school crime. [...] Mutual assistance, instead of teeing the most natural form of cooperation and association, becomes a clandestine effort to relieve one's neighbor of his proper duties. [...] Helping others, instead of being a form of charity which impoverishes the recipient, is simply an aid in setting free the powers and furthering the impulse of the one helped. A spirit of free communication, of interchange of ideas, suggestions, results, both successes and failures of previous experiences, becomes the dominating note of the recitation. [...] It is in the comparison of individuals, not with regard to the quantity of information personally absorbed, but with reference to the quality of work done - the genuine community standard of value. In an informal but all the more pervasive way, the school life organizes itself on a social basis.

(John Dewey, 1907, p. 29-30)

\section{Introducción}

Preocuparse por la calidad de un sistema educativo es, en definitiva, ocuparse de la cotidianeidad de su funcionamiento. Más allá de los resultados concretos de los alumnos, con interés específico para la comparación internacional (OECD, 2004), lo cierto es que los aspectos sociales y comunitarios en los que se desarrolla la acción educativa han atraído progresivamente la atención de los educadores y, especialmente, la de los investigadores interesados por una educación de calidad. Primero, desde la perspectiva de la eficacia escolar (Purkey y Smith, 1983, y Rowan, Bossert y Dwyer, 1983, para una revisión) y, más adelante, gracias al movimiento del estudio de los procesos para la mejora (Hopkins, Ainscow y West, 1994). Las últimas cuatro décadas de evolución 
de los estudios de calidad en la educación reflejan la toma de conciencia progresiva acerca de la relevancia de la incidencia de la cultura de funcionamiento de los centros en el rendimiento de los estudiantes (Lamperes, 2005; Hargreaves, 1995; Hargreaves, 1994).

Aspectos como la promoción y el desarrollo de una visión, de unos valores y de unos objetivos conjuntos (Stoll y Fink, 1994; Louis y Miles, 1990; Rosenholtz, 1989; Coleman y Hoffer, 1987; Cohen, 1983; Lightfoot, 1983), el establecimiento de normas para la colaboración (Blank, Melaville y Shah, 2003; McLaughlin, 1993; Little, 1982), el desarrollo de una estrategia de liderazgo participativo en torno a la promoción de la autonomía de todos los actores implicados (Sawyer, 2001; Wohlstetter, 1995; Lieberman, 1990; Stedman, 1987), el compromiso y la participación de los padres (Wholstetter y otros, 2001; Hickman, 1996; Hickman, Greenwood y Miller, 1995; Dornbusch y Ritter, 1988; Becher, 1984; Tizard, Schofield y Hewison, 1982), la promoción de las relaciones con el contexto local (Blank y otros, 2003; Wholstetter y otros, 2001; Louis y Miles, 1990; Coleman y Hoffer, 1987), la generación de un sentimiento de pertenencia (Goodenow, 1993; Mortimore y otros, 1988) o el propio desarrollo comunitario en el contexto de la escuela (Bryk y Schneider, 2002; Wholstetter y otros, 2001; Lee y otros, 1993; Bryk y Driscoll, 1988), han resultado ser claves para el óptimo funcionamiento de un sistema escolar.

Sin embargo, hablar del binomio formado por la escuela ${ }^{1}$ y la comunidad no es un asunto sencillo. Y, como hemos visto, no se trata de un asunto ajeno a las preocupaciones educativas. Al contrario, el término "comunidad» se ha extendido con gran éxito en el lenguaje educativo cotidiano, pero, como en el caso de los términos «sistema» o "cultura», lo ha hecho a costa de una pérdida sustancial de su contenido. Así, resulta habitual el uso de términos como «comunidad educativa» o "comunidad escolar», a pesar de que realmente resulta difícil encontrar en ellos los elementos definitorios del propio concepto de comunidad. Su uso, más bien, responde a la necesidad de hacer referencia al conjunto de actores que, respectivamente, comparten bien un espacio (el centro educativo no universitario), bien una función (la responsabilidad de educar, sin especificar en este caso el nivel educativo). Más allá de los matices que podamos establecer alrededor de este tipo de expresiones, lo cierto es que su (ab)uso cotidiano ha terminado por desgastar la propia idea original de comunidad hasta convertirla en un simple sinónimo de "grupo social».

Por esta razón, para aproximarnos al estudio del desarrollo comunitario escolar, comenzaremos por discutir brevemente el propio concepto de comunidad, para así situar teóricamente los fenómenos por los que nos interesamos en el contexto educativo. Hechas las consideraciones oportunas, exploraremos los principales resultados de los estudios empíricos desarrollados en el con-

1. En adelante, utilizaremos el término «escuela» como sinónimo de «centro educativo de educación no universitaria", sin perjuicio de que nos refiramos a centros educativos que imparten estudios de educación primaria, secundaria obligatoria, secundaria postobligatoria o cualquiera de las combinaciones posibles. 
texto de Cataluña, prestando especial atención al enfoque y al tipo de aproximación adoptada. A continuación, introduciremos el concepto de capital social como instrumento para el desarrollo de un modelo de análisis que nos permita aproximarnos a los diferentes contextos de desarrollo comunitario en el contexto educativo, para, finalmente, explorar los resultados empíricos a partir de los diferentes indicadores de desarrollo comunitario en la actividad cotidiana del sistema educativo no universitario de Cataluña.

\section{El concepto de comunidad: de la contigüidad espacial a la construcción del «nosotros»}

El concepto de comunidad, a pesar de ser uno de los más atractivos para la investigación de la sociología (Hodgett, 2003), es, a la vez, uno de los más dificultosos de definir, operativizar y estudiar (Cohen, 1985). Un concepto que, además de esconder una multiplicidad de sentidos en función de los intereses de quien lo utiliza (Smelser, 1967; Hillery, 1955), ha sido el objeto en torno al cual se han librado algunos de los debates más apasionantes de la sociología moderna.

Entre ellos, quizá el más evidente sería el que centra su atención en la tesis de la pérdida de la comunidad, lugar común en la literatura científica, donde se plantea lo impersonal, transitorio, volátil o fragmentado del último siglo y medio de (des)organización social (Wirth, 1938). A partir de los trabajos de autores clásicos como Tönnies, Durkheim, Simmel o Weber, esta línea de trabajo se plantea con mayor o menor intensidad la progresiva destrucción de las formas tradicionales de asociación frente a los procesos de urbanización e industrialización que comenzaron a desarrollarse a escala global en el tránsito del siglo XIX al XX (Sampson, 1999), llegando incluso hasta nuestros días a manos de autores tan mediáticos como el profesor Robert D. Putnam (1996 y 2002). Paradójicamente, la comunidad se ha perdido y se ha redescubierto muchas veces en el tiempo en que se ha desarrollado este debate (Wellman, 1979, para una revisión), de forma que es necesaria una cierta cautela, no sólo para evitar volver la mirada nostálgicamente hacia un tiempo comunitario dorado perdido o para la reinvención de viejas soluciones (Sampson, 1999), sino para poder contemplar, en nuestra definición, las transformaciones que los procesos comunitarios han sufrido en las últimas décadas (Wellman, 1998 y 2001, para una revisión).

En sus inicios, y siempre teniendo en cuenta la relativa falta de consenso en su conceptualización, la comunidad fue entendida ampliamente como un derivado de la interacción basada en la proximidad geográfica (Hillery, 1955). Una «unidad territorial» (Poplin, 1972), definida por límites físicos que encierran un espacio geográfico compartido donde florecerían, casi necesariamente, los lazos densos primarios de relación. Sin embargo, este componente topográfico sustancial fue evolucionando a medida que el desarrollo científico-técnico permitió la progresiva disolución de las distancias espaciales y temporales, mediante el desarrollo de los medios de transporte y especialmente los de comu- 
nicación (Wellman, 1998; Fischer, 1994; Fischer, 1982). Se abre un camino que, de acuerdo con Wellman (2001), transformará progresivamente la sociabilidad desde el «puerta a puerta», el «lugar a lugar»y, finalmente, el «persona a persona», haciendo compatibles y no excluyentes diferentes formas de comunidad. Como han señalado Lee y Newby (1983, p. 57), que las personas vivan próximas no implica necesariamente que tengan relación alguna, de forma que, como decimos, más que deteriorarse y desaparecer, la actividad social se ha liberado progresivamente de la necesidad de anclaje físico, poniendo como base la elección por afinidad más allá de la simple contigüidad (Wellman y Wellman, 1992).

Vistos los procesos comunitarios en perspectiva, el lugar, es decir, la (com)unión a través del territorio, no parece ser necesariamente la clave. Y menos aún si tenemos en cuenta las transformaciones que se están sucediendo en las últimas décadas. Pero, si no es así, ¿dónde podemos encontrar el mínimo común denominador para una definición del término "comunidad»?

George Hillery (1955), en su revisión de la literatura a mediados del siglo pasado, describió casi una centena de definiciones diferentes del concepto de comunidad. A pesar de que aproximadamente tres cuartas partes aún hacían uso de las barreras físicas para definir la propia comunidad, su conclusión fue que «todas las definiciones tienen que ver con la gente. Más allá de este punto en común, no hay acuerdo» (p. 117). Sin embargo, quizá esta aseveración pudiera resultar, a los ojos del lector, poco precisa e incluso en exceso restrictiva. Es cierto que los procesos comunitarios tienen que ver con las personas, pero, como se ha señalado más recientemente, quizá tengan que ver más bien con algún otro tipo de límite subyacente a tanta dispersión. Y quizá podamos encontrarlo, independientemente del origen, en la percepción de una identidad común, en la conciencia de la existencia de una serie de normas, creencias, valores y objetivos comunes.

Como señalaba Sennett (2001) al referirse a la dificultad de definición de este término y su confusión en el lenguaje popular, «comunidad es un término social engañoso. La gente habla de comunidad de intereses, por ejemplo, individuos que realizan la misma clase de trabajo o dependen unos de otros para ganar dinero. También hay comunidades de sentimientos, como iglesias o grupos étnicos, cuyos miembros poseen vínculos sentimentales entre sí" (p. 71). Asumimos, si es que llega a ser el caso, la existencia de una conciencia colectiva, de un sentimiento de identidad común, elementos que desde luego no resultan irrelevantes en la definición de los procesos comunitarios. Al menos si nos planteamos, como así pretendemos en esta investigación, un análisis riguroso y comprometido de la actividad comunitaria en el contexto escolar. «Una comunidad — proseguía el mismo Sennett (2001)— es una particular variedad de un grupo social en la que los hombres [y mujeres] creen que ellos comparten algo juntos. [...] El vínculo de comunidad es el de percibir identidad común, un placer en reconocernos a nosotros y a lo que somos» (p. 71).

Sin lugar a dudas, descubrir este reconocimiento mutuo en el uso común y cotidiano de los términos "comunidad escolar» o "comunidad educativa» 
como si de un ente abstracto se tratara, resulta una tarea cuando menos compleja. Por esta razón, como veremos, nuestro análisis aplicado al contexto educativo que presentamos a continuación no puede tener otro punto de partida que el de la consideración de la construcción explícita de esta percepción de unidad, más allá de su mera asunción bajo cualquier supuesto rasgo común.

\section{Una aproximación inicial al desarrollo comunitario del contexto escolar de Cataluña}

Hecha esta consideración inicial, necesaria para abordar con garantías una perspectiva comunitaria de la educación, ¿`cómo podríamos decir que se encuentra el sistema educativo no universitario catalán desde un punto de vista comunitario? Una primera forma de aproximación para responder a esta cuestión podría ser la de Orriols, Roldán y Oliart (2004). En un estudio desarrollado por el Institut d'Estadística de Catalunya en colaboración con el Departament d'Educació de la Generalitat, los autores nos ofrecen algunas cifras interesantes alrededor del estudio del papel de las asociaciones de madres y padres (AMPA, en adelante) en el conjunto de actividades y servicios complementarios desarrollados en los centros de Cataluña.

El supuesto, como en buena parte de los estudios comparativos de desarrollo comunitario, es el de que éste tiene un gran correlato en los niveles de afiliación y de participación en las estructuras de participación formal. La actividad comunitaria informal, la que podríamos convenir que es la crucial, resulta muy costosa de aprehender, de forma que la ventaja de la existencia de registros escritos en torno al asociacionismo convierte a esta forma particular de actividad en una de las fuentes principales para este tipo de estudios. Una comunidad fuerte, se asume desde esta posición, se caracterizaría, entre otras cosas, por un importante desarrollo del tejido asociativo. Por el contrario, un desarrollo más limitado de este tejido podría ser un indicador de la escasa capacidad para la colaboración entre los diferentes implicados. Trasladando este razonamiento al contexto educativo, el estudio de los indicadores de participación formal podría tomar como punto de partida la presencia y el grado de implicación en el desarrollo de las actividades de los centros por parte de las AMPA, las asociaciones por excelencia del contexto educativo no universitario.

De esta forma, de acuerdo con los datos que ofrece este estudio, correspondientes al curso escolar 2001-2002, las AMPA estarían presentes en algo más de un $85 \%$ de los centros, dando cobertura con sus servicios a algo más del 95\% de los alumnos inscritos en los centros educativos no universitarios de Cataluña. Estas cifras, referidas únicamente a la escuela pública, se incrementan sensiblemente cuando no consideramos los centros de atención de 0 a 3 años. En comparación con etapas superiores del sistema educativo, la presencia de las AMPA en estos centros no superaría el 50\%, muy probablemente por la dificultad que supone una permanencia breve en el centro para la generación del tejido asociativo. 
Por otro lado, al considerar estos datos globalmente, sabemos que su presencia en la gestión de las actividades de los centros se circunscribiría fundamentalmente al área de las actividades educativas complementarias (57\%) y al «suministro» de libros de texto (43\%). Dos de los tres servicios complementarios más realizados en los centros de Cataluña y de los que se ocupan casi en exclusividad, con una presencia prácticamente testimonial de las direcciones de los centros y ayuntamientos. En el caso del comedor escolar, el servicio más frecuente, a la vez que más importante desde el punto de vista de gasto económico, la responsabilidad queda repartida uniformemente. Los ayuntamientos o consejos comarcales destacan ligeramente en este aspecto, de hecho, es donde ejercen su papel más destacado.

Si bien esta aproximación podría ayudarnos a comprender el panorama de organización de las actividades complementarias y, específicamente, el papel de las AMPA como vehículo de éstas, también es cierto que la participación formal es sólo una pequeña parte del amplio puzzle de la actividad comunitaria. Por esta razón, en un intento por conseguir una perspectiva más amplia, resulta muy interesante la consideración de la propuesta del Institut de Govern i Polítiques Públiques de la Universitat Autònoma de Barcelona. El estudio de Alsinet, Riba, Ribera y Subirats (2003), desarrollado en colaboración con el Institut de Ciències de l'Educació de la misma universidad y la Fundació Jaume Bofill, analiza la actividad comunitaria de las escuelas de Cataluña, a partir del estudio de su participación en proyectos desarrollados en su entorno comunitario más inmediato.

En esta ocasión, la investigación se centra en el estudio de las «experiencias educativas integradas", es decir, aquellas intervenciones educativas comunitarias en donde diferentes agentes del entorno de la escuela (autoridades educativas, entidades locales, empresarios y comerciantes, padres, sindicatos, asociaciones de vecinos, etc.) participan junto al centro mismo en la detección y solución de problemas educativos. Para ello, a través de la red de centros de recursos pedagógicos (CRP) del Departament d'Educació de la Generalitat, se puso en marcha una búsqueda de experiencias en activo que cumplieran estos requisitos, obteniendo un censo de algo más de sesenta. El análisis de los autores, basado en el estudio cualitativo de los seis casos más significativos de acuerdo con los objetivos de investigación, nos ofrece también algunas cifras que deberíamos considerar en el estudio del desarrollo comunitario de las escuelas de Cataluña.

Así, aún a riesgo de perder los detalles de la exploración cualitativa, podemos decir que, en general, la mayoría de los proyectos analizados en el 2002 tienen que ver con la búsqueda de nuevas maneras para «afrontar los nuevos problemas y las nuevas necesidades que plantea la sociedad actual» (Alsinet y otros, 2003, p. 61), tanto en las esferas familiar, como social y productiva. Además, aproximadamente la mitad de las sesenta y siete experiencias recogidas hacen referencia a proyectos cuyos objetivos pretenden la reducción del riesgo de marginación social de los escolares provenientes de familias con ingresos económicos bajos, especialmente alrededor de la reducción de la desmotivación, 
el fracaso escolar, el absentismo y la violencia en los centros, las drogadicciones y la falta de hábitos saludables para la prevención, entre otros.

Ahora bien, a pesar de que el número de proyectos y el tipo de problemáticas que tratan de afrontar parecen mostrar una interesante implicación de la escuela en el desarrollo de la intervención comunitaria, lo cierto es que, al abordar cuestiones como la antigüedad, la implicación de las administraciones o el grado de estructuración de los propios proyectos, no podemos dejar de considerar estas cifras con cautela. Si bien podemos encontrar algunos proyectos con más de un lustro de duración, resultan más bien excepcionales en comparación a los iniciados entre 1997 y 2002 (81\%). Por otro lado, no más de un $40 \%$ del total de los proyectos se encuentra en una fase aceptable de desarrollo, donde tanto los objetivos y los instrumentos, como la planificación y el seguimiento, están claramente definidos. En este caso, cuando disponen de una estructuración razonable, la gran mayoría cuenta, además, con la participación de al menos una de las administraciones implicadas en Cataluña (y, en casos muy concretos, los propios centros), lo que desempeña un importante papel en su impulso y desarrollo. En el resto, bien por la ausencia de objetivos, bien por tratarse realmente de primeras experiencias donde aún no existen instrumentos para acometerlas, la ausencia de implicación institucional dificulta aún más si cabe la generación de respuestas integradas.

\section{La generación de capital social en el sistema educativo no universitario}

Las dos formas de aproximación a los fenómenos comunitarios que hemos visto, tanto a través del análisis de la actividad impulsada por las AMPA como del desarrollo de los "proyectos educativos integrados», serían ejemplos de lo que algunos autores han venido llamando "capital social». A continuación, antes de presentar un modelo de análisis del desarrollo comunitario centrado en la actividad cotidiana de los centros educativos no universitarios, introduciremos brevemente el concepto de capital social.

\subsection{El concepto de capital social en el estudio de los fenómenos comunitarios}

Este concepto, acuñado por Lyda Judson Hanifan $^{2}$ en Estados Unidos a principios del siglo pasado, pretendía hacer referencia inicialmente a la importancia de la participación comunitaria para el correcto funcionamiento de las escuelas. Inspector educativo en el contexto rural, Hanifan (1916) propuso por primera vez este uso metafórico del concepto de capital para reflejar precisamente la bondad o el valor — a imagen y semejanza de otras formas de

2. Farr (2004) para una interesante arqueología conceptual que permite trazar las bases sobre las que el propio Hanifan pudo desarrollar el concepto, situando a Dewey en el verdadero origen, tanto por su utilización anterior de la expresión «capital social» en un texto escrito, como por las similitudes entre la visión de la educación del propio Dewey y la perspectiva comunitaria adoptada por Hanifan en su actividad como inspector educativo. 
capital bien conocidas - de las propias relaciones sociales como oportunidades para establecer colaboración en beneficio mutuo. Pero, como se desprende de su relato en el contexto escolar de principios del siglo XX, no sólo haría referencia al beneficio desde el punto de vista individual de los implicados directos en los espacios de colaboración, sino considerando el beneficio que la comunidad, en su conjunto, obtendría a través del desarrollo de estrategias cooperativas por parte de sus componentes.

Esta perspectiva, que se nutre fundamentalmente de las aportaciones teóricas desarrolladas en torno a una aproximación grupal o colectiva ${ }^{3}$, lo define por encima de los matices entre los diferentes autores como el conjunto de redes y normas para la colaboración en beneficio mutuo (entre otros, Putnam, 1993 y 2002; Cohen y Prusak, 2001; OECD, 2001; Fukuyama, 2000; Woolcock y Narayan, 2000; Coleman, 1988). A pesar de que, en algunas ocasiones, esta perspectiva ha sido criticada por una excesiva imprecisión en la definición (véase, por ejemplo, Coleman, 1988, p. 98) o por la consecuente ausencia de acuerdo acerca de la consideración de la confianza, bien como causa, bien como consecuencia (véase, por ejemplo, Putnam, 2002, p. 14), el consenso a partir de la creciente evidencia empírica ha permitido la acotación «en sus fuentes más que en sus consecuencias» (Woolcock, 2001, p. 13). Sólo así ha sido posible la operativización del concepto de capital social, donde, ahora sí, la confianza ha pasado a ser considerada una consecuencia y, por tanto, un indicador, junto a la participación formal en asociaciones, la participación política o la actividad social informal.

$\mathrm{Si}$, como decíamos más arriba, el concepto de comunidad ha sido una de las áreas estrella de la investigación en sociología, su reformulación al abrigo de la teoría del capital social no está siendo menos para la investigación social contemporánea (Woolcock, 2003). Y ello está totalmente justificado, ya que, gracias a su extraordinaria ductilidad como marco teórico, no sólo ha sabido hacerse un hueco en el propio lenguaje de la sociología, sino que también ha permitido desarrollar múltiples propuestas en disciplinas como la economía, la política, la medicina, el urbanismo, la epidemiología, la psicología social y comunitaria, o la educación entre otras (por ejemplo, ver Putnam, 2002, para una extensa recopilación de los principales resultados en un amplio abanico de disciplinas). Sin embargo, como señala Woolcock (2001), no se trata simplemente de «un buen marketing para viejas ideas» (p. 13), sino de un nuevo lenguaje que nos permite avanzar en una misma dirección, reduciendo la oscuridad de la jerga académica con que a veces los científicos sociales se han aproximado a estos fenómenos.

Independientemente de la aproximación en la definición, la idea fundamental gira entorno a la importancia de las relaciones sociales que indicába-

3. Entre otros, Robison, Schmid y Siles (2002), Lin (2001), Baker (2000), Portes (1998), Burt (1992) o Bourdieu (1985), para una perspectiva alternativa individual fundamentada en la evaluación de los recursos a los que los individuos pueden acceder en virtud de las relaciones sociales. 
mos más arriba. Si el estudio etimológico del término "comunidad» nos lo revela compuesto originariamente a partir de la yuxtaposición de las palabras cum (preposición que significa 'con, juntos, conjuntamente') y munus (sustantivo que significa 'servicio, regalo, favor'), el interés por las relaciones sociales y la obtención de beneficios (tanto individuales o directos, como grupales o indirectos) a través del análisis del capital social, ha permitido establecer una importante y fructuosa línea de investigación transdisciplinaria y comparada (Field, 2003; Ostrom y Ahn, 2003; Putnam, 2003; Robison y Flora, 2003; Fukuyama, 2000; Portes, 1998; Putnam, 2002, para una extensa revisión).

Sin embargo, y centrándonos específicamente en el contexto escolar que ahora nos ocupa, los indicadores que hemos observado a partir de los estudios considerados más arriba quizá no puedan dar cuenta de estos fenómenos con la riqueza que desearíamos. Permiten que podamos hacernos una idea inicial de dos aspectos importantes para este tipo de estudios, pero no podemos perder de vista que estos procesos se caracterizan por una complejidad mucho mayor de la que se puede aprehender a través de un análisis exclusivamente centrado en indicadores formales. Al contrario, para conocer con detalle el grado de desarrollo comunitario de nuestro sistema educativo, es necesario que nos aproximemos a los espacios de colaboración donde pueden generarse los procesos de construcción del nosotros a que antes aludíamos, teniendo bien presentes a todos y cada uno de los actores implicados en la actividad cotidiana de la escuela. Dicho de otro modo, un análisis en profundidad del desarrollo comunitario del sistema educativo requiere complementar la información procedente de los estudios basados en indicadores formales, profundizando en el análisis de las oportunidades para el encuentro, la interacción y la colaboración, tanto en el aula como en el contexto organizacional de los centros educativos.

\subsection{Un modelo analítico basado en la teoría del capital social para el estudio del desarrollo comunitario en la actividad cotidiana escolar}

Para construir un modelo de análisis que nos permita profundizar en el conocimiento del nivel de desarrollo comunitario, de acuerdo con el planteamiento defendido hasta el momento, podríamos considerar como punto de partida la propuesta de Anthony P. Cohen en su magnífica obra La construcción simbólica de la comunidad. Cohen (1985), preguntándose cuáles pueden ser las bases de la generación de la comunidad, nos ofrece las claves para una aproximación procesual, anclada en la actividad cotidiana, que nos permite entrever cómo es posible aproximarnos al desarrollo del sentimiento de comunidad o de pertenencia en un grupo determinado a partir de la actividad social. Teniendo en cuenta, ante todo, que la creación de comunidad es un proceso fundamentalmente simbólico, la base común a cualquiera de ellas se encontraría precisamente en la capacidad de un grupo humano para acordar y mantener la creencia de que: a) sus componentes tienen algo en común entre ellos, que b) los distingue significativamente de otros grupos de adopción. Sin embargo, 
esta percepción construida a partir del establecimiento de normas y valores compartidos que sirven de límites o barreras simbólicas, tal y como la estamos desarrollando en el contexto escolar, no debería suponer necesariamente la negación de los otros-que-no-son-nosotros. Más bien al contrario, la constatación de que existen en sí mismos, como interlocutores distintos de nosotros mismos.

Desde esta perspectiva, como veremos más adelante, tan importante resulta para un análisis de la actividad comunitaria la capacidad de establecer el nosotros como de reconocer el vosotros, en tanto interlocutores para la colaboración en beneficio mutuo. De forma consecuente, desde el contexto local donde la escuela se encuentra hasta el nivel del aula donde se desarrolla la práctica pedagógica, tan importantes resultan para la creación de comunidad las oportunidades generadas en el contexto interno de los centros escolares, como la interacción con los agentes del contexto externo en el que se inserta. Tan relevante es el desarrollo de colectivos en la escuela con intereses y objetivos compartidos, como su participación en las dinámicas de gestión del centro. Tan significativa resulta la estimulación de una práctica docente basada en la promoción del trabajo en equipo, como la disposición del profesorado a colaborar con madres y padres, profesionales, asociaciones, empresas o incluso con otros centros educativos del contexto.

Una noción relacional de comunidad donde los procesos de control social son entendidos, más que como una forma de represión o conformidad, como la capacidad de un colectivo para gestionar la heterogeneidad individual de intereses, valores y objetivos, creando no sólo unidad a partir de la diversidad (Erickson, 1988), sino también su organización efectiva en la busca de objetivos comunes en beneficio mutuo (Janowitz, 1975; Kasarda y Janowitz, 1974). Lo importante, como apuntaba Wellman (1979), es determinar la existencia de las redes que generan pertenencia, evitando asumir su organización local espacialmente determinada, para así poder contemplar las nuevas posibilidades que se han generado en el último siglo (p. 1202-1203). Sólo así es posible el abordaje del concepto de comunidad, evitando la conclusión errónea de su pérdida por no desarrollarse necesariamente en el marco de la contigüidad espacial que ha venido siendo normativo en la larga historia de las relaciones sociales.

Así pues, para afrontar con garantías la exploración de todas estas cuestiones en el contexto del sistema educativo no universitario, estructuraremos nuestro análisis en torno a la consideración de dos tipos fundamentales de indicadores, tratando de ofrecer algo de luz en los principales ámbitos de actividad cotidiana de los centros. Dos tipos que surgen precisamente de la distinción introducida por Gittel y Vidal (1998) entre capital social vinculante (o inclusivo) y capital social que tiende puentes (o exclusivo). Esta distinción, emparentada conceptualmente con el análisis de la exclusión social y la pobreza de Briggs (1998 y 2001) y de la fuerza de los lazos débiles de Granovetter (1973 y 2003), ha resultado de una fertilidad tal que rápidamente ha trascendido las áreas específicas en las que tuvo su origen para formar parte del aparataje conceptual propio del capital social (Grootaert, Narayan, Jones y Woolcock, 2004; 
Larsen y otros, 2004; Field, 2003; Putnam, 2002 y 2003; Stone y Hughes, 2002; Lin, 2001; Woolcock, 2001; Woolcock y Narayan, 2000; Burt, 1992).

Desde este punto de vista, las diversas expresiones de capital social podrían ser clasificadas en, al menos, dos grupos fundamentales: capital social vinculante y capital social que tiende puentes. Por un lado, el vinculante (del inglés bonding, es decir, que une, que pega, que ata, que vincula), se haría cargo de la interacción que se produce entre individuos similares, lo que conduciría a la homogeneidad en la relación, entre componentes de grupos de referencia establecidos a partir de aspectos importantes como la etnia, el sexo, la edad, la clase social, etc. Un primer tipo de actividad social imprescindible para la generación de un nosotros a partir del desarrollo de lazos fuertes que permitan una estrecha colaboración en grupos relativamente homogéneos ante demandas y objetivos semejantes. Es el caso, por ejemplo, de la promoción de la cooperación como estrategia de trabajo en el aula, el desarrollo de estrategias para el trabajo en equipo entre el profesorado del mismo curso o etapa, así como del establecimiento de los mecanismos necesarios para facilitar la participación de los colectivos internos en la gestión del centro como organización.

En el otro extremo de la dimensión, y siempre teniendo presente que, como en toda distribución dimensional, los tipos puros no suelen ser los más frecuentes, el capital social que tiende puentes (del inglés bridging, es decir, que llena huecos, que reduce distancias entre puntos no comunicados por otros medios), se haría cargo de la interacción menos frecuente entre individuos diferentes, lo que conduciría a la heterogeneidad en la relación. La clave, como el propio Granovetter finalmente concluye, no está necesariamente en la debilidad de los lazos en sí misma, sino en que «tienden en una gran proporción a ser puentes cuando se les compara con los fuertes, que apenas tienen esta cualidad» (Granovetter, 2003, p. 227). Un segundo tipo de actividad social que permite la colaboración, la implicación y la corresponsabilización de los otrosque-no-son-nosotros. Hablamos en esta ocasión, por ejemplo, de la disposición del profesorado a la participación de agentes de la comunidad en la dinámica del aula, del desarrollo y la participación en redes de colaboración a través de proyectos educativos compartidos entre centros, así como el establecimiento de espacios y vías para la corresponsabilización de los agentes de la comunidad local en la dinámica de los centros.

Sin embargo, y ya para finalizar, algo que se debe desprender de esta distinción esencial es que ni ambos tipos son intercambiables, ni hay un único responsable del adecuado funcionamiento de un colectivo. Como se ha mostrado en la literatura (Larsen y otros, 2004; Warren, Thompson y Saegert, 2001; Woolcock y Narayan, 2000; Ferguson y Dickens, 1999; Sampson, Raudenbush y Earls, 1997; Putnam, 1993; Sampson, 1988), el desarrollo comunitario óptimo comienza por la formación de un fuerte sustrato de capital social vinculante, donde los lazos sólidos entre los componentes permiten la organización en busca de objetivos comunes por encima de la heterogeneidad de intereses. Establecida esta condición inicial, la clave para el desarrollo de la acción conjunta estaría muy relacionada, tanto con la relativización de 
las diferencias entre los distintos grupos o colectivos, como con la implicación de organizaciones e instituciones externas a través del desarrollo del capital social que tiende puentes. Este tipo de actividad, por su capacidad para servir de puentes entre grupos densos de interacción frecuente, adquiere entonces una enorme relevancia (Wellman, 1979 y 2001) al margen de la importancia que tradicionalmente había sido atribuida en los estudios clásicos comunitarios al establecimiento de redes densas de relación de fuerte compromiso (por ejemplo, entre otros, Tönnies, 1977; Wirth, 1938; Nisbet, 1969).

Recuperando la noción de comunidad sobre la construcción del nosotros que sosteníamos más arriba, la clave no resulta ser su mera construcción a partir de unas normas y valores compartidos que sirvan de límites o de barreras simbólicas excluyentes con respecto a los otros-que-no-son-nosotros (generación de capital social vinculante), sino a través de la constatación contingente de su existencia como interlocutores válidos en un marco más amplio de colaboración en beneficio mutuo (generación de capital social que tiende puentes), más allá de los propios límites del entramado de interacciones densas y frecuentes.

Desde esta doble distinción fundamental, nuestra exploración de indicadores de actividad social y comunitaria en el entorno educativo no universitario, de creación de comunidad, se estructura alrededor de los dos principales contextos de actividad cotidiana escolar: una perspectiva de la práctica pedagógica, en torno al contexto del aula, así como una perspectiva organizacional, focalizando la atención ahora en el centro en su conjunto como contexto de actividad. La tabla 1 representa esquemáticamente ambos tipos de relaciones, así como el tipo de fenómenos que exploraremos para estudiar el desarrollo comunitario a partir de la creación de los diferentes nosotros en la actividad escolar cotidiana.

Tabla 1. Modelo analítico para el estudio del binomio formado por la escuela y la comunidad.

\begin{tabular}{lll}
\hline & Capital social vinculante & $\begin{array}{l}\text { Capital social } \\
\text { que tiende puentes }\end{array}$ \\
\hline $\begin{array}{l}\text { Perspectiva de la } \\
\text { práctica pedagógica } \\
\text { (el aula como contexto) }\end{array}$ & $\begin{array}{l}\text { Colaboración del alumnado } \\
\text { en la dinámica del aula. }\end{array}$ & $\begin{array}{l}\text { Colaboración entre } \\
\text { centros en proyectos } \\
\text { educativos compartidos. } \\
\text { Participación de } \\
\text { colectivos externos en la } \\
\text { dinámica del aula. }\end{array}$ \\
\hline $\begin{array}{l}\text { Perspectiva } \\
\text { organizacional } \\
\text { (el centro como }\end{array}$ & Colaboración del profesorado \\
contexto) & $\begin{array}{l}\text { Participación } \\
\text { de colectivos externos } \\
\text { en la dinámica }\end{array}$ \\
& $\begin{array}{l}\text { Participación docente. } \\
\text { internos en la dinámica }\end{array}$ & \\
\hline
\end{tabular}

Fuente: Elaboración propia. 
$\mathrm{O}$, dicho de otro modo, intentaremos responder a algunas cuestiones como ¿hasta qué punto disponen los alumnos de espacios para la colaboración dentro de la dinámica del aula? ¿Participan nuestros centros en proyectos educativos compartidos? ¿Qué tipo de relación establecen nuestras aulas con la comunidad local y los diversos agentes externos implicados en la educación? ¿Trabajan los profesores en colaboración para alcanzar conjuntamente los objetivos educativos? ¿Qué medidas se están disponiendo desde las direcciones de los centros para que esta colaboración sea efectiva? ¿Cuentan con sensibilidad para la aportación de los diferentes colectivos implicados en la toma de decisiones del propio centro? ¿Qué incidencia tienen en la dinámica del centro las madres y los padres, a través de su relación individual con la dirección o el profesorado, más allá del radio de acción de las AMPA? ¿Y el resto de colectivos del contexto local?

\section{Metodología}

Para responder a las cuestiones planteadas a partir del modelo analítico, nuestro estudio explora cada uno de los ámbitos de construcción de comunidad a partir de los datos obtenidos en el Projecte Internet Catalunya: L'escola a la Societat Xarxa (Mominó, Sigalés, Fornieles, Guasch y Espasa, 2004), desarrollado por el Internet Interdisciplinary Instute (IN3) de la Universitat Oberta de Catalunya (UOC), con la financiación de la Generalitat de Catalunya y la colaboración del Departament d'Educació y de la Fundació Jaume Bofill.

Este proyecto de investigación, dirigido por los profesores Carles Sigalés y Josep Maria Mominó, pretende identificar y analizar la incorporación de Internet a los centros educativos de Cataluña (educación primaria y secundaria), observando en qué transformaciones está presente y en qué medida contribuye a la aparición de una nueva cultura educativa adaptada a las necesidades que se van configurando en la sociedad informacional. Sin embargo, el interés por un análisis complejo que considere el funcionamiento de los centros educativos, nos ha permitido acercarnos, a través de este proyecto de investigación, a muchos otros aspectos de la actividad cotidiana del sistema educativo no universitario de forma complementaria. Uno de ellos, el desarrollo comunitario, es el que en esta investigación nos ocupa, y del que ahora trataremos de hacer una pequeña aproximación presentando los resultados principales.

Por tanto, el universo de estudio está compuesto por el alumnado, el profesorado y los equipos directivos de los 2.726 centros con sede en Cataluña (España) donde se imparten estudios de educación primaria, educación secundaria obligatoria (ESO), bachillerato y ciclos formativos de grado medio (CFGM) o superior, durante el curso 2002-2003 de acuerdo con las estadísticas facilitadas por el Departament d'Educació de la Generalitat de Catalunya. Teniendo en cuenta este universo, se fija el tamaño muestral en 350 centros aceptando un margen de error de $\pm 5 \%$ en los resultados globales de los centros, un nivel de confianza del $95,5 \%$ con el supuesto de máxima indeterminación $(p=q=0,50 \mathrm{y} k=2)$. Con el objetivo de obtener la máxima representatividad ante la diversidad del universo, el diseño muestral fue aleatorio, 
estratificado y multietápico, teniendo en cuenta las siguientes variables: niveles o ciclos formativos, distribución territorial, ámbito rural / urbano y titularidad pública / privada.

De esta forma, los resultados que aquí presentamos se basan en la información recogida a través de una encuesta compuesta por cinco cuestionarios, administrada a una muestra representativa de todos los centros docentes no universitarios de Cataluña (España) a través de una entrevista personal con cada uno de los actores implicados en el funcionamiento de las escuelas. Así, para cada uno de los centros seleccionados en la muestra, se entrevistó al director, al responsable pedagógico de la etapa seleccionada, al responsable de TIC del centro, así como al conjunto de alumnos y su profesorado de un grupo clase elegido al azar del último curso de la etapa seleccionada. De forma complementaria, una ficha de centro permitió también la recogida de todos los datos referidos a la institución.

El trabajo de campo fue realizado entre los meses de diciembre de 2002 y abril de 2003 por un equipo de encuestadores formados específicamente en la administración de estos cuestionarios mediante entrevista personal a un total de 6.612 alumnos (2.918 de primaria, 1.883 de secundaria obligatoria, 1.269 de bachiller y 542 de CFGM o superior), 2.163 profesores (785 de primaria, 673 de secundaria obligatoria, 533 de bachillerato y 127 de CFGM o superior) y 1.050 responsables de los equipos directivos (525 de primaria, $246 \mathrm{de}$ secundaria obligatoria, 177 de bachillerato y 102 de CFGM o superior).

\section{Resultados}

Siguiendo el modelo de análisis que acabamos de plantear (ver tabla 1), exploraremos a continuación los diferentes espacios para el desarrollo comunitario, de acuerdo tanto con los dos tipos de capital social (vinculante y que tiende puentes), como con los diferentes contextos de actividad cotidiana de la educación primaria y secundaria: la perspectiva de la práctica pedagógica, considerando la actividad en el aula como contexto de generación de capital social, $\mathrm{y}$, a continuación, la perspectiva de la organización, atendiendo a las oportunidades que los propios centros como organizaciones ofrecen para estos fines.

\subsection{El desarrollo comunitario desde la perspectiva de la práctica pedagógica: el aula como contexto de generación de capital social}

El aula, eje central de la actividad educativa formal, es la primera parada en nuestro análisis de la actividad comunitaria de los centros de Cataluña ${ }^{4}$. Una actividad en el aula que, como decíamos, contemplaría diferentes entramados de relaciones para alcanzar los objetivos de la acción docente. Desde este

4. Mominó y Meneses (2006) para una revisión en profundidad de la actividad docente en clave pedagógica. 
punto de vista, la creación de comunidad en nuestros centros partiría, en primer lugar, del análisis del grado en que la dinámica del aula esté basada en una estrategia de trabajo en equipo de los propios alumnos entre sí, promocionando la colaboración entre iguales, más directamente relacionados con el desarrollo de una pertenencia - de un nosotros - anclada en la cotidianeidad del aula. Donde el trabajo individual y la competencia son progresivamente sustituidos por un modelo de corresponsabilización, de ayuda mutua, de colaboración, donde la interacción densa y frecuente sirve de base para la consecución de objetivos y requerimientos similares. Un modelo que transforma la figura del profesor en un experto que acompaña en el proceso de aprendizaje y a los alumnos en aprendices activos que desarrollan su actividad en un marco de interacción social del aula (Marchesi y Martín, 1998, para una revisión de la investigación reciente).

Observando, pues, en primer lugar, las formas de trabajo que se están promoviendo en las aulas, sabemos que la cultura del trabajo en equipo en el aula no es la más extendida a pesar de la importancia que tradicionalmente se le ha concedido al abandono de prácticas educativas más individualistas, directivas o conservadoras. Examinando las políticas de centro, de acuerdo con la información proporcionada por los equipos directivos, este tipo de trabajo en el aula resulta estar bastante o muy presente en la propuesta educativa de dos tercios de los centros educativos $(62,1 \%)$. Sin embargo, a pesar de la relevancia que tiene en términos globales, cuando nos interesamos por el grado de aplicación de este objetivo, un 70,4\% del total de los centros se encuentra implementándolo o en un grado avanzado de implementación. Solamente uno de cada diez centros $(10,7 \%)$ tendría este objetivo conseguido. De esta forma, cuando descendemos a la práctica en el aula, algo más de un tercio del profesorado $(34,2 \%)$ considera que, en su actividad, el tipo de trabajo promovido entre los alumnos es más bien individual. De hecho, apenas una quinta del total $(21,3 \%)$ considera que su actividad está bastante o muy centrada en una estrategia de cooperación, éstos últimos - los que promueven el trabajo en equipo de forma habitual- no suponiendo más de un 3,4\% del total.

Por otro lado, un segundo espacio de actividad cotidiana interesante para un análisis como éste es el del grado en que las escuelas catalanas colaboran entre ellas para el desarrollo de proyectos educativos conjuntos. Lamentablemente, los aspectos compartidos entre centros en muchas ocasiones se deben seguramente más a su relación común con la administración, en una estructura de organización fundamentalmente radial del sistema educativo, que a su interconexión en el contexto geográfico próximo. Esta situación no solo plantea una dificultad para la identificación de la especificidad local en los retos y problemas de cada escuela (Gairín, 2005), sino que deja, a su vez, muy poco margen a las iniciativas basadas en la coordinación entre centros del mismo territorio. Es cierto que, gracias a este modelo de gestión del sistema educativo, se ha alcanzado una cierta autonomía en el funcionamiento interno de las escuelas, pero la escasez de oportunidades para el encuentro entre ellas también ha dificultado enormemente el desarrollo de políticas conjuntas 
de colaboración interorganizacional basada en la coincidencia en preocupaciones, problemas, retos y, por tanto, objetivos.

Centrando nuestro interés ahora en la participación y el desarrollo de proyectos educativos conjuntos entre centros, y de acuerdo con la importancia que concede el profesorado a este tipo de colaboración, hemos podido comprobar que son muy pocos $(5,9 \%)$ los que consideran que generalmente no compensa la inversión de esfuerzo requerida para llevarlos a cabo. Por el contrario, una amplia mayoría (94\%) considera que, o bien pueden ser interesantes en circunstancias muy concretas $(64,1 \%)$, o bien resultan experiencias en las que es muy importante que participen profesores y alumnos (29,9\%). Sin embargo, cuando tratamos de determinar la implicación efectiva del profesorado del sistema educativo no universitario catalán, el escenario no resulta tan favorable: aproximadamente dos tercios de las escuelas $(65,6 \%)$ habitualmente no colabora con otros centros en el desarrollo de proyectos educativos compartidos. Consecuentemente, el profesorado que participa de forma habitual en este tipo de proyectos $(6,2 \%)$ supone realmente una excepción, y aunque si bien es cierto que un amplio sector lo hace de forma ocasional $(43,5 \%)$, resulta significativo descubrir que aproximadamente la mitad $(50,2 \%)$ nunca, ni siquiera de forma esporádica, participa en este tipo de proyectos.

Finalmente, en tercer lugar, desde un planteamiento como el que hacemos nos interesaría la promoción de una dinámica de trabajo que saque provecho también de aquellos entramados de relaciones que permiten la colaboración con los agentes y los colectivos del contexto local, más directamente relacionados con el reconocimiento de la importancia de su participación —-del reconocimiento de un vosotros - en la actividad cotidiana del aula. Un modelo que trasforme a la educación en un asunto de interés colectivo, y a la escuela en un agente educativo más, quizá el que asume una responsabilidad directa mayor, pero al fin y al cabo uno más (Subirats, 2002, para un análisis del sistema educativo español en unos términos similares). Una visión sobre la propia educación donde la responsabilidad educativa recaiga especialmente en los mecanismos descritos por los profesionales de la escuela, pero teniendo en cuenta la relevancia de la corresponsabilización en el proceso de los diferentes agentes y colectivos implicados en el contexto local a través de la apertura del aula a la participación de colectivos externos como madres y padres, profesionales, asociaciones, empresas, administraciones, etc. Colectivos bien diferenciados y en muchos casos considerados ajenos a la cuestión educativa, heterogéneos en cuanto a sus intereses y objetivos con respecto a la escuela, que, sin embargo, deben ser considerados a partir de su contribución en la forma de interacciones de una frecuencia e implicación menores.

La cuestión, como decíamos, no está únicamente en garantizar unos adecuados niveles de trabajo cooperativo entre el alumnado como estrategia de trabajo. Es necesaria también la consideración del grado de apertura con que la dinámica del aula responde a la influencia de los agentes y colectivos externos. Lamentablemente, desde este punto de vista, las perspectivas no resultan mucho mejores. Al contrario, cuando nos aproximamos a la percepción de los 
profesores sobre la participación externa en su actividad docente, son realmente pocos los que indican una colaboración habitual con los padres de los alumnos $(14,4 \%)$ o con profesionales e instituciones de fuera de la escuela $(13,7 \%)$. Y es que resulta casi anecdótica la existencia de docentes que consideren que su práctica está preferentemente organizada alrededor de la participación de otros agentes externos. Solamente un $7,8 \%$ considera que su actividad en el aula está bastante o muy orientada a este tipo de participación, mientras que, en el otro extremo, aproximadamente dos tercios del total $(67,4 \%)$ indica que la actividad en el aula está fundamental o completamente al margen de esta influencia.

\subsection{El desarrollo comunitario desde la perspectiva organizacional: el centro como contexto de generación de capital social}

Observados los aspectos principales concernientes a una práctica pedagógica sensible a los aspectos sociales y educativos, nos aproximaremos a continuación a la segunda perspectiva de análisis: el desarrollo comunitario de las escuelas de Cataluña desde una perspectiva organizacional.

De acuerdo con la tabla 1, para conocer el desarrollo comunitario con el propio centro como contexto de actividad cotidiana, debemos comenzar por conocer hasta qué punto el trabajo en equipo y la participación en las decisiones del centro forman parte de la cultura de funcionamiento en el día a día. A pesar de que, desde el punto de vista de la descentralización, las competencias en materia educativa no han alcanzado aún el nivel de la Administración local, los centros educativos no universitarios tienen un cierto espacio para organizar su funcionamiento de forma autónoma. De hecho, por mucho que se trate de un sistema educativo altamente centralizado y con unidad de planteamientos, las escuelas son organizaciones que siempre «acaban configurando realidades educativas diferentes, consecuencia directa del filtro que la historia institucional y la cultura dominante dan a cualquier influencia externa» (Gairín, 1999, p. 236-237). Hablamos de una cultura o de un clima que, como ocurre en multitud de organizaciones ajenas al contexto escolar, no sólo tiene una influencia determinante en su funcionamiento global, sino que también se conforma a través de las relaciones cotidianas entre todos sus componentes.

Así, comenzaremos por referirnos a los indicadores relativos al tipo de trabajo desarrollado por el profesorado como colectivo (Antúnez, 1999), donde la colaboración entre los docentes del mismo centro se establecería entorno a su relación densa y frecuente alrededor de procedimientos y objetivos similares. De acuerdo con la información proporcionada por el profesorado, dos de cada tres docentes (64\%) indican que el trabajo en equipo es una estrategia habitual en su actividad profesional fuera del aula. Esta práctica, que contrasta notablemente con apenas el $2 \%$ que no trabaja nunca en colaboración con su equipo, parece ser además una de las claves en las políticas de los centros, de manera que la planificación de objetivos compartidos que permitan su trabajo en colaboración resulta ser un aspecto prioritario para algo más de la mitad 
de los responsables de etapa entrevistados (55,9\%). De hecho, teniendo en cuenta las diez prioridades principales para las direcciones de los centros, entre las que podemos encontrar, por ejemplo, el establecimiento de sistemas para mejorar el aprovechamiento del tiempo, de sistemas innovadores para atender a la diversidad, la formación del profesorado, la mejora de los sistemas de comunicación o la promoción de procesos de evaluación del centro, únicamente el establecimiento de objetivos que permitan mejorar el funcionamiento del centro resulta ser una prioridad más extendida que el fomento de la cooperación.

Por otro lado, de acuerdo con el segundo tipo de relaciones, tomando el centro como contexto de actividad cotidiana, exploraremos ahora el grado y las formas de participación que los diferentes actores implicados en el contexto interno de la escuela (profesorado, dirección, alumnos, etc.) tienen en su funcionamiento, donde la colaboración se establece ahora en torno a la convergencia de los propios intereses en busca del establecimiento de una cultura de gestión compartida de la cotidianeidad del centro como organización. Más allá de los mecanismos formales de representación, disponemos de alguna información acerca de los tipos de participación impulsada por los equipos directivos, así como de la implicación efectiva del profesorado.

Así, desde la óptica de los directores de los centros, la participación del alumnado se centraría fundamentalmente en cuestiones organizativas, más que aquéllas relacionadas con su propio proceso educativo. Dos tercios de los directores consideran que la participación de los alumnos es efectiva en aspectos como la decisión de las actividades a desarrollar en el centro $(70,8 \%)$ o la elaboración de normas de convivencia y funcionamiento (69,9\%). Sin embargo, como decíamos, esta proporción desciende notablemente cuando nos interesamos por su implicación en las decisiones en aspectos relacionados con su aprendizaje, como, por ejemplo, su propio proceso de evaluación $(48,2 \%)$ o la definición de su propio ritmo de aprendizaje (42\%). Por el contrario, de acuerdo con las direcciones de los centros, la participación del profesorado queda circunscrita, mayoritariamente, a los procesos de organización del centro $(96,3 \%)$, así como a cuestiones estrictamente académicas, como el desarrollo del proyecto educativo de centro $(95,4 \%)$ o la incorporación de las TIC e Internet $(81,7 \%)$. Sin embargo, al contrario que en el caso del rol esperado de los alumnos, la implicación en las cuestiones administrativas o económicas $(50,9 \%)$ queda en un discreto segundo plano.

Un patrón diferencial emerge entre profesores y alumnos, muy probablemente de acuerdo con lo que tradicionalmente se ha esperado de cada uno de ellos desde una concepción más tradicional de la dirección del centro, donde la participación, y especialmente la relacionada con la toma de decisiones sobre el proceso educativo, queda en mayor medida reservada para el propio colectivo del profesorado. Sin embargo, más allá de la emergencia de este patrón, resulta también interesante contrastar estos niveles de implicación con la participación que el propio profesorado informa estar desarrollando. De acuerdo con su criterio, matizando a la baja la perspectiva ofrecida por la dirección, 
únicamente uno de cada tres profesores $(31,7 \%)$ dice participar habitualmente en las decisiones que se toman en su centro. En comparación con el trabajo en colaboración con sus equipos respectivos, este nivel de participación supone un descenso de un tercio del total del profesorado, mientras que se incrementa notablemente el número de docentes que nunca participa en estos procesos (15,3\% del total).

Finalmente, para acabar nuestra exploración de los diversos espacios para la construcción del nosotros de los centros educativos no universitarios de Cataluña, nos referiremos ahora brevemente a los mecanismos que se desarrollan desde el centro para promover su vinculación con el territorio próximo, con la comunidad local a la que pertenece. Si bien desde la perspectiva pedagógica nos interesamos por el nivel de apertura del aula al contexto local, desde un punto de vista más amplio, nuestro objetivo sería conocer el tipo de implicación que el conjunto de agentes de la comunidad local tiene en el funcionamiento del centro. Se trataría de colectivos bien diferenciados, como las propias AMPA, empresas, asociaciones, etc., heterogéneos en cuanto a sus intereses y objetivos con respecto a la escuela, a través de interacciones de una frecuencia e implicación menores.

Como señalaba Subirats (2002), el anclaje local, es decir, la importancia de la comunidad y el territorio en la modulación del papel de la escuela en nuestra sociedad, es un asunto relativamente novedoso con mucho espacio para su desarrollo. Más allá de las reformas necesarias del marco regulador que promuevan la implicación de la administración local, al menos la idea de que las escuelas no deben funcionar al margen de la comunidad en la que desarrollan su proyecto educativo forma parte del imaginario acerca de cómo debe funcionar una escuela. El desarrollo de las asociaciones de madres y padres, la organización de actividades extraescolares e incluso la colaboración con otras asociaciones y empresas ajenas inicialmente al contexto escolar, son instrumentos de corresponsabilización que, con mayor o menor éxito, han servido en las últimas décadas para facilitar la apertura de los centros educativos a su contexto.

Si bien es cierto que, para una gran mayoría de las escuelas $(95,7 \%)$, la colaboración ha resultado habitual con los servicios de soporte educativo como los equipos de atención primaria (EAP), los centros de recursos pedagógicos (CRP) o los servicios sociales, no podemos decir lo mismo con respecto al mundo asociativo y empresarial. En este caso, la proporción de escuelas que colaboran habitualmente desciende sensible y progresivamente hasta un 57,8\% de los centros que colaboran habitualmente con fundaciones, asociaciones y ONG, mientras que no se acercan a la mitad (44,2\%) los centros que hacen lo propio con empresas. Sin embargo, un capítulo especial por su vinculación como miembros de la comunidad local, es el de la relación de las madres y los padres con la escuela. A pesar de que una gran parte de las relaciones entre la familia y la escuela vienen encauzadas por la relación directa y privada con los profesores responsables de la educación de sus hijas e hijos, sería conveniente también tener en cuenta su implicación en los procesos de gestión que trascienden al aula. 
Para ello, más allá de los indicadores de participación formal de las AMPA que hemos introducido al comienzo de nuestra exploración, las impresiones que las direcciones de los centros ofrecen, constatan que en tres cuartas partes $(73,6 \%)$ la participación de este colectivo es habitual cuando nos referimos a la organización de actividades extraescolares. Sin embargo, esta proporción se reduce sensiblemente al hablar de decisiones relativas al currículum: si bien en algo menos de la mitad $(41,9 \%)$ las madres y los padres participan en los procesos de evaluación de los propios centros, son realmente una excepción $(8,5 \%)$ aquéllos en los están implicados en las decisiones curriculares. De hecho, cuando nos detenemos en la naturaleza de la participación a que nos estamos refiriendo, podemos constatar cómo la atención de los padres y las madres se dirige de forma prioritaria a funciones que podríamos considerar tradicionales, quedando en segundo término las demandas vinculadas de forma más directa a la innovación. De esta forma, la mitad de los directores $(51,5 \%)$ aprecian una concentración de las demandas en lo referido a los procesos de transmisión de conocimientos, mientras que, ya sólo una cuarta parte $(27,2 \%)$, constata la preocupación por cuestiones disciplinarias. Aspectos como la incorporación de la informática a la formación $(10,7 \%)$, el aprendizaje de idiomas $(8,3 \%)$ o el uso de Internet (3\%), resultan ser los más alejados del centro de atención.

\section{Discusión}

Como hemos podido ver, el concepto de capital social tiene una profunda raigambre en el contexto educativo. Pero no únicamente porque una de las primeras pruebas escritas de su uso hayan quedado asociadas a Hanifan en el contexto de las escuelas rurales norteamericanas de principios del siglo $\mathrm{XX}$, sino por la indispensable aportación que muchos otros autores clásicos en la teoría del capital social, como Bourdieu, Coleman o Putnam, han venido produciendo desde el contexto de la escuela o de la educación en general (Dika y Singh, 2002, para una de las escasas revisiones de la literatura en este ámbito). Sin embargo, y a pesar del interés que hemos constatado por los fenómenos comunitarios en la investigación sobre calidad en la acción educativa (Lamperes, 2005; Hargreaves, 1995; Hargreaves, 1994; Hargreaves, 2000, para una discusión acerca de la importancia de las redes para el desarrollo de la educación en la sociedad de la información), son más bien una excepción los estudios que, como el que presentamos en este artículo, persiguen un análisis de la actividad comunitaria en el contexto escolar a partir del estudio de los diferentes indicadores de colaboración en la actividad cotidiana.

De este caso, del análisis concreto del desarrollo comunitario en el contexto educativo no universitario de Cataluña, podemos extraer algunas conclusiones. En primer lugar, y de acuerdo con el planteamiento de nuestro modelo, la comunidad es una cuestión simbólica, una percepción de identidad compartida construida a partir de las redes y de las normas para la colaboración generadas en los diferentes contextos de actividad cotidiana de las escuelas. 
Más que un fenómeno cuya existencia debamos dar por supuesta, es una determinada manera de entender la educación, tanto la actividad pedagógica propiamente dicha como su organización, donde poner en práctica los beneficios de la acción conjunta, tanto en el contexto del aula como del propio centro.

Sin embargo, los niveles de desarrollo son bastante diferentes. Si bien existe un cierto interés por estas cuestiones a nivel del aula, aún es difícil concluir que la promoción del trabajo en equipo, la participación en proyectos educativos con otros centros o la apertura del aula a la participación de los diferentes agentes y colectivos de la comunidad en la que se integra la escuela sean las estrategias más extendidas en la actualidad. Al contrario, más allá de los intereses abstractos y de las políticas, los indicadores ligados a la práctica pedagógica cotidiana parecen mostrar al aula como un espacio poco desarrollado para estos fines, donde no son muchas las oportunidades para la acividad comunitaria.

Por su parte, el centro educativo como organización, es uno de los espacios clásicos para el desarrollo de la actividad comunitaria. En este sentido, los indicadores de participación y trabajo en equipo nos han mostrado como existe una cierta cultura de la colaboración, sobre todo si detenemos nuestro análisis en las formas de trabajo desarrolladas por el profesorado como colectivo. En cambio, en lo referente a la participación, aún podemos encontrar espacios para el desarrollo de nuevas oportunidades para la generación del nosotros. Si bien se espera una cierta complicidad de los colectivos internos y externos, hemos podido comprobar cómo emergen patrones diferenciales donde los tipos de participación esperados son más próximos a formas más tradicionales de gestión de los centros.

\section{A modo de conclusión: el desarrollo comunitario como fin y no meramente como medio}

Explorados los ámbitos más interesantes de construcción de comunidad en el contexto no universitario, más allá de las posibles valoraciones acerca de su grado de desarrollo, convendría hacer una pequeña reflexión final acerca de la importancia de la actividad comunitaria, no sólo como medio, sino también como fin en sí misma para el éxito de la educación de nuestros alumnos en las escuelas.

La adopción de una perspectiva preocupada por la creación de comunidad en el contexto educativo, no es simplemente una cuestión de mejora del rendimiento. Es cierto que los movimientos interesados por la calidad en la educación, como la Eficacia Escolar y del Estudio de los Procesos para la Mejora, se han centrado inicialmente en este objetivo, pero la cuestión no se dirime exclusivamente en este terreno. Una escuela interesada en asumir un proyecto integral de funcionamiento comunitario es, en realidad, aquélla que tiene como objetivo último la preocupación por hacer significativa la experiencia educativa. Aspectos como el fomento del sentimiento de pertenencia, la participación, la corresponsabilización, el trabajo en equipo o el acercamiento a la comuni- 
dad local nos hablan de calidad en los procesos, de la construcción del nosotros en los diferentes espacios disponibles, no únicamente del resultado. De esta forma, por ejemplo, el propio proyecto PISA, la apuesta de la OCDE para la evaluación de la calidad en la educación internacional a partir del rendimiento de los estudiantes, se hace eco también de algunos de estos aspectos en sus análisis (Willms, 2003). Sustituyendo el propio rendimiento por medidas de vinculación y pertenencia, la lectura de Willms de los resultados del PISA plantea el éxito de los sistemas educativos por su capacidad para implicar a los estudiantes y, por ende, por su participación efectiva en el funcionamiento cotidiano del aula y del propio centro.

Ya sea en la dinámica de trabajo de las aulas, en la forma que tienen los propios centros para organizar su funcionamiento o en la apertura al entorno de ambos contextos en los que se desarrolla la acción educativa, una perspectiva favorable al desarrollo de la comunidad constituye, en este sentido, una forma imprescindible cuando se trata de resolver con eficacia los retos y los problemas diarios de la escuela. Una estrategia que, haciendo partícipes y responsables a sus miembros en cada uno de los distintos espacios, se plantea extraer el máximo provecho de la colaboración en beneficio mutuo. Sería el caso, como un ejemplo muy interesante para ilustrar este punto, del programa cooperativo para el fomento de la reutilización de libros de texto y material didáctico complementario del Departament d'Educació de la Generalitat de Catalunya. Una iniciativa que pretende la regulación y dotación de recursos a los proyectos de socialización de libros que han ido emergiendo de forma independiente en los últimos años.

Tímidamente, y gracias al impulso de las AMPA, en algunas escuelas de Cataluña ha surgido en los últimos años el interés por resolver uno de los problemas más antiguos de nuestra escolarización obligatoria: el coste del material escolar. Tras años de discusión acerca de su gratuidad, o al menos la reducción significativa de su coste, algunas escuelas parecen haber encontrado la clave en la colaboración para resolver el problema. Y lo han hecho a través de un proyecto que podríamos enclavar en el centro de los fenómenos de interés que hemos explorado en este artículo. Mediante un sistema autogestionado por cada centro, con apoyo de la Generalitat desde el curso 2005-2006, estos proyectos de socialización de recursos se basan fundamentalmente en la necesidad de reutilizar los libros de texto y el material educativo complementario. Pero no de forma individual, como se venía haciendo en el seno de las familias con varios niños, sino de forma coordinada en el contexto del aula, bajo la supervisión de una comisión en la que participan todos los colectivos implicados en la escuela. A través de una pequeña cuota anual, y sobre todo gracias a la reutilización del parque de libros de texto en circulación, el objetivo es convertir al alumno - y la familia, al fin y al cabo - en usufructuario del material indispensable para su educación.

Sin embargo, no podemos agotar sus fines en el terreno meramente económico. Más allá del beneficio individual que puede suponer no tener que comprar libros nuevos cada curso, la filosofía de fondo se basa en el fomento 
del valor de la cooperación, la implicación y la corresponsabilización para hacer un uso racional y sostenible de los recursos. La colaboración, desde el punto de vista comunitario, no es únicamente un medio, es a la vez un fin. Una aproximación radicalmente diferente a la propuesta de la subvención total o parcial del coste de los libros que sabe capitalizar el éxito económico final en un cambio en la actividad cotidiana de los alumnos. Los libros, a partir de ahora, no tienen otro dueño que la escuela. Y el alumno, más que mero usuario del material educativo como soporte a su educación, resulta ser el responsable en la escuela de expandir su uso, velando porque su vida útil no se agote en sus propias manos.

Al fin y al cabo, el fomento de la colaboración en beneficio mutuo es, al mismo tiempo, algo más que un simple instrumento para facilitar nuestros objetivos. Es una finalidad en sí misma, la forma en que podemos concretar los valores de una educación comprometida, justa, responsable, participativa y solidaria. Tenida en cuenta la reducción en el gasto familiar que supone la socialización de los recursos, este programa pretende también reducir las desigualdades económicas en el acceso a los recursos educativos, el fomento de la responsabilidad de los alumnos sobre el material, la promoción de una cultura de reciclaje y reutilización de los recursos, el desarrollo de la capacidad para compartir, colaborar y respetar el bien común, así como la corresponsabilización de los diferentes colectivos implicados en la dinámica del centro, haciendo especial hincapié en la gestión autónoma por parte de la comunidad educativa (Generalitat de Catalunya, 2005).

Un magnífico ejemplo de cómo las escuelas son más que las organizaciones en las que los niños aprenden conocimientos y habilidades (Dewey, 1907, p. 29-30). Son, como señalaba Etzioni (1993), «lugares en donde se adquiere - o no se consigue adquirir- educación» (p. 248). Una educación que incluye la puesta en práctica y el refuerzo de los valores aprendidos en casa o, en el peor de los casos, la enseñanza de éstos para aquéllos cuyos padres no supieron o no pudieron hacerlo. De ahí la importancia de los fenómenos comunitarios, sin lugar a dudas, y de la necesidad de continuar fomentando los espacios de colaboración en beneficio mutuo que faciliten su desarrollo en la actividad cotidiana de nuestras escuelas. Especialmente si, además, lo que pretendemos es plantearnos la calidad como una seria aspiración para nuestra educación, el futuro mismo de nuestra sociedad.

\section{Agradecimientos}

Este trabajo ha sido posible gracias al apoyo del Departament d'Universitats, Recerca i Societat de la Informació de la Generalitat de Catalunya y del Fondo Social Europeo. 


\section{Referencias bibliográficas}

Alsinet, J.; Riba, C.; Ribera, M.; Subirats, J. (2003). Més enllà de l'escola: Transformacios socials $i$ noves dinàmiques educatives $i$ professionals. Barcelona: Mediterrània.

ANTÚNEZ, S. (1999). "El trabajo en equipo de los profesores y profesoras: factor de calidad, necesidad y problema. El papel de los directivos escolares». Educar, 24, 89-110.

BAKER, W. (2000). Achieving success through social capital: Tapping the hidden resources in your personal and business networks. Nueva York: Jossey-Bass.

BECHER, R. M. (1984). Parent involvement: a review of research and principles of successful practice. Urbana (IL): ERIC Clearinghouse on Elementary and Early Childhood Education.

Blank, M. J.; MelaVIlle, A.; SHAH, B. P. (2003). Making the difference: research and practice in Community Schools. Retrieved November 1st, 2005, from Coalition for Community Schools Website: <http://www.communityschools.org/CCSFull Report.pdf>.

Bourdieu, P. (1985). «The forms of capital». En: RicharSON, J. G. (ed.). Handbook of theory and research for the Sociology of Education. Nueva York: Greenwood.

BRIGGS, X. de S. (1998). "Brown kids in white suburbs: housing mobility and the many faces of social capital». Housing Policy Debate, 9(1), 177-221.

- (2001). Ties that bind, bridge and constrain: social capital and segregation in the american metropolis (versión electrónica). Comunicación presentada en el International Seminar on Segregation and the City, Cambridge, 26 al 28 de julio.

BRYK, A. S.; DrISCOLL, M. E. (1988). The high school as community: contextual influences and consequences for students and teachers. Madison: University of WisconsinMadison, National Center on Effective Secondary Schools.

BRYK, A. S.; SCHNEIDER, B. (2002). Trust in Schools. A core resource for improvement. Nueva York: Russell Sage Foundation.

BURT, R. S. (1992). Structural holes, the social structure of competition. Cambridge (MA): Harvard University Press.

CoHen, A. P. (1985). The symbolic construction of community. Londres: Routledge.

COHEN, D.; PRUSAK, L. (2001). In good company. How social capital makes organizations work. Boston (MA): Harvard Business School Press.

COHEN, M. (1983). «Instructional, management and social conditions in Effective Schools». En: ODDEN, A.; DEAN, L. (eds.). School finance and school improvement linkages for the 1980s. Cambridge (MA): Ballinger, p. 17-50.

Coleman, J. S. (1988). «Social capital in the creation of human capital». American Journal of Sociology, 94, 95-121.

Coleman, J. S.; HofFer, T. (1987). Public and private high schools: the impact of communities. Nueva York: Basic Books, Inc.

DEWEY, J. (1907). The School and society: being three lectures by John Dewey supplemented by a statement of the University Elementary School. Chicago: University of Chicago Press.

DIKA, S. L.; SINGH, K. (2002). «Applications of social capital in educational literature: a critical synthesis». Review of Educational Research, 72(1), 31-60.

DORnBusCH, S. M.; RitTER, P. L. (1988). «Parents of high school students: a neglected resource». Educational Horizons, 66, 75-77. 
ERICKSON, B. H. (1988). «The relational basis of attitudes». En: Wellman, B.; BERKOWITZ, S. (eds.). Social structures: a network approach. Nueva York: Cambridge University Press, p. 99-121.

ETZIONI, A. (1993). The spirit of community. The reinvention of American society. Nueva York: Touchstone.

Ferguson, R. F.; Dickens, W. T. (1999). «Introduction». En: Ferguson, R. F.; DiCKENS, W. T. (eds.). Urban problems and community development. Washington, D.C.: Brookings Institution Press, p. 1-31.

FIELD, J. (2003). Social capital. Londres: Routledge.

FISCHER, C. S. (1982). To dwell among friends: personal networks in town and city. Chicago: The University of Chicago Press.

- (1994). America Calling. A social history of the telephone to 1940. Berkeley: University of California Press.

FuKUYAMA, F. (2000). La gran ruptura. Naturaleza humana y reconstrucción del orden social. Barcelona: Ediciones B.

GAIRÍN, J. (1999). La organización escolar: contexto y texto de actuación (2a ed.). Madrid: Editorial la Muralla.

- (2005). La descentralización educativa: ¿una solución o un problema? Barcelona: Praxis.

Generalitat de Catalunya (2005). Programa cooperatiu per al foment de la reutilització de llibres de text $i$ material didàctic complementari. Retrieved November 1st, 2005, from Departament d'Educació Website, <http://www.gencat.net/ense/ beques/beq11.htm>.

GITTEL, R.; VIDAL, A. (1998). Community organizing. Building social capital as a development strategy. Thousand Oaks: Sage Publications.

GoODENOW, C. (1993). «The psychological sense of school membership among adolescents: Scale development and educational correlates». Psychology in the Schools, 30, 79-90.

GranOVETTER, M. S. (1973). "The strength of weak ties». American Journal of Sociology, 78, 1360-1380.

- (2003). «La fuerza de los lazos débiles. Revisión de la teoría reticular». En: REQUENA, F. (ed.). Análisis de redes sociales: Orígenes, teorías y aplicaciones. Madrid: Centro de Investigaciones Sociológicas, p. 196-230.

Grootaert, C.; NARAyan, D.; Jones, V. N.; WoOlcock, M. (2004). «Measuring social capital: an integrated questionnaire». World Bank Working Paper Series, 18. Retrieved November 1st, 2005, from PovertyNet Library Website: <http://poverty2.forumone.com/files/11998_WP18-Web.pdf>.

HaNifan, L. J. (1916). «The rural school community center». Annals of the American Academy of Political and Social Science, 67, 130-138.

HARGREAVES, A. (1994). Changing teachers, changing times. Teacher's work and culture in the postmodern age. Londres: Cassell.

HARGREAVES, D. (1995). «School culture, school effectiveness and school improvement». School effectiveness and school improvement, 6(1), 23-46.

- (2000). «Lessons for education: creating a learning system». En: OECD (ed.). Knowledge management in the learning society. París: Centre for Educational Research and Innovation, p. 67-96.

HiCKMAN, C. W. (1996). «The future of high school success: the importance of parent involvement programs». Special issue of The High School Journal. Retrieved November 1st, 2005, from Horizon Website: <http://horizon.unc.edu/projects/HSJ/ Hickman.asp>. 
Hickman, C. W.; Greenwood, G. E.; Miller, M. D. (1995). «High school parent involvement: relationships with achievement, grade level, SES, and gender». Journal of Research and Development in Education, 28, 125-134.

HillerY, G. (1955). "Definitions of community: areas of agreement». Rural Sociology, 20, 111-123.

Hodgett, S. L. (2003). «Sense of community». En: Christensen, K.; LevinSON, D. (eds.). Encyclopedia of community. From the village to the virtual world, vol. I. Thousand Oaks: Sage Publications, p. 236-238.

HOPKINS, D.; AINSCOW, M.; WEST, M. (1994). School improvement in an era of change. Londres: Casell.

JANOWITZ, M. (1975). «Social theory and social control». American Journal of Sociology, 81(1), 82-108.

KaSARDA, J. D.; JanOWITZ, M. (1974). "Community Attachment in Mass Society». American Sociological Review, 39, 328-39.

LAMPERES, B. (2005). Making change happen: shared vision, no limits. Lanham (MD): Scarecrow Education.

Larsen, L.; Harlan, S. L.; Bolin, B.; Hackett, E. J.; Hope, D.; Kirby, A.; Nelson, A.; ReX, T. R.; Wolf, S. (2004). «Bonding and bridging. Understanding the relatioship between social capital and civil action». Journal of Planning Education and Research, 24, 64-77.

LeE, D.; NewBy, H. (1983). The problem of sociology: an introduction to the discipline. Londres: Unwin Hyman.

LEE, V.; BRYK, A.; SMITH, J. (1993). «The organization of effective secondary schools». En: Darling-Hammond, L. (ed.). Review of Research in Education, 19, p. 171-226.

LiEBERMAN, A. (1990). Schools as collaborative cultures: Creating the future now. Nueva York: The Falmer Press.

LightFOOT, S. L. (1983). The good high school: Portraits of character and culture. Nueva York: Basic Books.

Lin, N. (2001). Social capital. A theory of social structure and action. Cambridge: Cambridge University Press.

LiTTLE, J. W. (1982). «Norms of collegiality and experimentation: workplace conditions of school success». American Educational Research Journal, 19(3), 325-340.

LOUIS, K. S.; MILES, M. B. (1990). Improving the urban high school: what works and why. Nueva York: Teachers College Press.

MARCHESI, A.; MARTÍn, E. (1998). «El alumno y la construcción del conocimiento». En: MARCheSI, A.; MARTín, E. (eds.). Calidad de la enseñanza en tiempos de cambio. Madrid: Alianza Editorial, p. 323-352.

MCLaughlin, M. W. (1993). "What matters most in teachers' workplace context». En: LitTle, J. W.; MCLAughlin, M. W. (eds.). Teachers' work. Individuals, colleagues, and contexts. Nueva York: Teachers College Press, p. 51-76.

MOMINÓ, J. M.; MENESES, J. (2006). «¿Una práctica pedagógica para la Sociedad Red? Un análisis de la acción docente en el ámbito educativo no universitario». Teoria de la Educación: Educación y Cultura en la Sociedad de la Información, 7(1), Retrieved April 1st, 2006, from TE Website: <http://www3.usal.es/-teoriaeducacion/ rev_numero_07/n7_art_momino_meneses.htm $>$.

Mominó, J. M.; Sigalés, C.; Fornieles, A.; GuAsCh, T.; Espasa, A. (2004). L'escola a la Societat Xarxa: internet en l'àmbit educatiu no universitari. Informe de recerca. Retrieved November 1st, 2005, from Internet Interdisciplinary Institute Website: $<$ http://www.uoc.edu/in3/pic/cat/pic31.html . 
Mortimore, P.; SAmmons, P.; STOLl, L.; LeWIS, D.; ECOB, R. (1988). School matters: the junior years. Somerset: Open Books.

NisBeT, R. (1969). The quest for community. Nueva York: Oxford University Press.

OECD (2001). The wellbeing of nations. The role of human and social capital. París: Center for Educational Research and Innovation.

- (2004). Learning for Tomorrow's World. First Results from PISA 2003. París: Center for Educational Research and Innovation.

Ostrom, E.; Ahn, T. K. (2003). Foundations of social capital. Cheltenham (UK): Edward Elgar Publishing.

OrRiols, P.; RoldÁn, E.; Oliart, J. (2004). «Los servicios y actividades complementarias en la educación pública: Una aproximación empírica al papel de las asociaciones de madres y padres de alumnos en Cataluña». Índice: Revista de Estadística y Sociedad, 8, 18-19.

Poplin, D. E. (1972). Communities: a survey of theories and methods of research. Nueva York: Macmillan.

PORTES, A. (1998). «Social capital: its origins and applications in modern sociology». Annual Review of Sociology, 24, 1-24.

PURKEY, S.; SMITH, M. (1983). «Effective schools: a review». Elementary School Journal, 83(4), 426-452.

Putnam, R. D. (1996). «The strange disappearance of civic America». American Prospect, 7(24). Retrieved November 1st, 2005, from TAP Website: <http://www.pros pect.org/print/V7/24/putnam-r.html>.

- (2002). Solo en la bolera: Colapso y resurgimiento de la comunidad norteamericana. Barcelona: Galaxia Gutenberg.

- (2003). El declive del capital social. Un estudio internacional sobre las sociedades y el sentido comunitario. Barcelona: Galaxia Gutenberg.

RoBISON, L. J.; FLORA, J. L. (2003). «The social capital paradigm: bridging across disciplines». American Journal of Agricultural Economics, 85(5), 1187-1193.

Robinson, L. J.; SCHMID, A. A.; SiLES, M.E. (2002). «Is social capital really capital?». Review of Social Economy, 60(1), 1-21.

ROSENHOLTZ, S. J. (1989). Teachers' workplace: the social organization of schools. Nueva York: Teachers College Press.

ROWAN, B.; BOSSERT, S. T.; DWYER, D. C. (1983). «Research on effective schools: A cautionary note». Educational Researcher, 12 (4), 24-31.

SAMPSON, R. J. (1988). «Local friendship ties and community attachment in mass society: a multilevel systemic model». American Sociological Review, 53, 766-779.

— (1999). "What "community" supplies». En: Ferguson, R. F.; Dickens, W. T. (eds.). Urban problems and community development. Washington, D.C.: Brookings Institution Press, p. 241-279.

SAMPSON, R. J.; Raudenbush, S.; EARLS, F. (1997). «Neighborhoods and violent crime: a multilevel study of collective efficacy». Science, 277, 918-924.

SAWYER, R. D. (2001). «Teachers who grow as collaborative leaders: the rocky road of support». Education Policy Analysis Archives, 9(38). Retrieved November 1st, 2005, from EPAA Website: <http://epaa.asu.edu/epaa/v9n38.html>.

SennetT, R. (2001). Vida urbana e identidad personal. Barcelona: Ediciones Península. SMELSER, N. J. (1967). Sociología. Madrid: Euramérica.

STEDMAN, L. C. (1987). "It's time we changed the effective schools formula». Phi Delta Kappan, 69(3), 215-227. 
STOLL, L.; FinK, D. (1994). «Views from the field: linking school effectiveness and school improvement». School Effectiveness and School Improvement, 5(2), 149-177.

STONE, W.; Hughes, J. (2002). Social Capital. Empirical meaning and measurement validity. Australian Institute of Family Studies. Research paper 27. Retrieved November 1st, 2005, from AIFS Website: <http://www.aifs.gov.au/ institute/pubs/RP27.pdf>.

SUBIRATS, J. (2002). Gobierno local y educación: La importancia del territorio y la comunidad en el papel de la escuela. Barcelona: Ariel Social.

TiZARD, J.; SCHOFIELD, W. N.; HEWISON, J. (1982). «Collaboration between teachers and parents in assisting children's reading». British Journal of Educational Psychology, $52,1-15$.

TÖnNies, F. (1977). Comunidad y sociedad. Buenos Aires: Losada.

WARREN, M. R.; THOMPSON, J. P.; SAEGERT, S. (2001). «The role of social capital in combating poverty». En: SAEGERT, S.; THOMPSON, J. P.; WARREN, M. R. (eds.). Social capital and poor communities. Nueva York: Russel Sage Foundation, p. 1-28.

Wellman, B. (1979). «The community question: the intimate networks of East Yorkers». American Journal of Sociology, 84(5), 1201-1231.

- (1998). "The network community. An introduction». En: Wellman, B. (ed.). Networks in the global village: life in contemporary communities. Boulder (CO): Westview Press, p. 1-48.

- (2001). "Physical place and cyberplace: the rise of personalized networking». International Journal of Urban and Regional Research 25(2), 227-52.

Wellman, B.; Wellman, B. (1992). «Domestic affairs and network relations». Journal of Social and Personal Relationships, 9, 385-409.

WILLMS, J. D. (2003). Student engagement at school. A sense of belonging and participation. Results from PISA 2000. Retrieved November 1st, 2005, from OECD Website: <http://www.pisa.oecd.org/dataoecd/42/35/33689437.pdf>.

WIRTH, L. (1938). «Urbanism as a way of life». American Journal of Sociology, 44, 3-24. WoOlCOCK, M. (2001). «The place of social capital in understanding social and economic outcomes». ISUMA: Canadian Journal of Policy Research, 2(1). Retrieved November 1st, 2005, from ISUMA Website: <http://www.isuma.net/v02n01/woolcock/woolcock_e.pdf>.

- (2003). «Social capital». En: Christensen, K.; Levinson, D. (eds.). Encyclopedia of community. From the village to the virtual world, vol. 3. Thousand Oaks: Sage Publications, p. 1258-1262.

WOOLCOCK, M.; NARAYAN, D. (2000). «Social capital: implications for development theory, research and policy». The World Bank Research Observer, 15(2), 225-249.

WOHLSTETTER, P. (1995). "Getting school-based management right: what works and what doesn't». Phi Delta Kappan, 77(1), 22-24, 26.

Wohlstetter, P.; Smith, A. K.; POlHemus, J. L.; HaO, L. (2001). Anatomy of School Family networks: a collaborative approach to reform. External evaluation report of the Los Angeles Annenberg Project. Retrieved November 1st, 2005, from University of Southern California Website: <http://www.usc.edu/dept/education/cegov/publi cations/laamp2001.pdf>. 